\title{
Vagus nerve stimulation balanced disrupted default-mode network and salience network in a postsurgical epileptic patient
}

This article was published in the following Dove Press journal:

Neuropsychiatric Disease and Treatment

II October 2016

Number of times this article has been viewed

\section{Kailiang Wang ${ }^{1,2}$ \\ Qi Chai' \\ Hui Qiao' \\ Jianguo Zhang ${ }^{2,3}$ \\ Tinghong Liu', ${ }^{1,2}$ \\ Fangang Meng ${ }^{1,2}$}

'Beijing Neurosurgical Institute, Capital Medical University, ${ }^{2}$ Beijing Key Laboratory of Neurostimulation, ${ }^{3}$ Department of Neurosurgery, Beijing Tiantan Hospital, Capital Medical University, Beijing, People's Republic of China
Correspondence: Fangang Meng Beijing Neurosurgical Institute, Capital Medical University, 6 Tiantan Xili, Dongcheng, Beijing 100050, People's Republic of China

Tel +861067096767

Fax +86 I067057507

Email fgmeng@ccmu.edu.cn
Introduction: In recent years, treatment of intractable epilepsy has become more challenging, due to an increase in resistance to antiepileptic drugs, as well as diminished success following resection surgery. Here, we present the case of a 19-year old epileptic patient who received vagus nerve stimulation (VNS) following unsuccessful left parietal-occipital lesion-resection surgery, with results indicating an approximate $50 \%$ reduction in seizure frequency and a much longer seizure-free interictal phase.

Materials and methods: Using resting-state functional magnetic resonance imaging, we measured the changes in resting-state brain networks between pre-VNS treatment and 6 months post-VNS, from the perspective of regional and global variations, using regional homogeneity and large-scale functional connectives (seeding posterior cingulate cortex and anterior cingulate cortex), respectively.

Results: After 6 months of VNS therapy, the resting-state brain networks were slightly reorganized in regional homogeneity, mainly in large-scale functional connectivity, where excessive activation of the salience network was suppressed, while at the same time the suppressed default-mode network was activated.

Conclusion: With regard to resting-state brain networks, we propose a hypothesis based on this single case study that VNS acts on intractable epilepsy by modulating the balance between salience and default-mode networks through the integral hub of the anterior cingulate cortex.

Keywords: vagus nerve stimulation, epilepsy monitoring, epilepsy surgery, functional neuroimaging, brain-network balance

\section{Introduction}

An intractable-epilepsy patient is generally considered to be one who shows little response to antiepileptic drugs (AEDs) and resection surgery, or those patients not suitable for surgical resection. ${ }^{1-4}$ Fortunately, vagus nerve stimulation (VNS) is a palliative therapy for refractory epilepsy approved by the US Food and Drug Administration for patients above the age of 12 years, and it has been reported to be both effective and safe. ${ }^{5-8}$ However, the mechanisms of VNS in intractable epilepsy are not fully known. Here, we introduce an intractable epilepsy case in which the patient received VNS after an unsuccessful seizure-controlled resection surgery, and obtained more than a $50 \%$ reduction in seizure frequency and a much longer seizure-free interictal phase after 6 months of treatment.

In recent years, studies have indicated that intractable epilepsy is not a focal but rather a network disease. ${ }^{9-11}$ Additionally, a number of studies have shown that intractable epilepsy impairs the organizing ability of resting-state networks of the brain, ${ }^{12-15}$ 
which are reflected in the coherent fluctuations in neuronal and neurovascular oscillations and their dynamic changes, as measured by blood oxygenation level-dependent (BOLD) signal power using functional magnetic resonance imaging (fMRI). ${ }^{16,17}$ Among these networks, the default-mode network (DMN) and salience network (SN) have been reported to have closer associations with the generation and propagation of abnormal epileptic activity that disrupts the dynamic interactions between networks and hence gives rise to the disorder. ${ }^{18-22}$ In the present study, we aimed to explore the functional mechanisms of VNS therapy in resting-state networks anchored in the DMN and SN, and we hypothesized that VNS therapy may relieve the epileptic symptoms by modulating the DMN and SN. In addition, we measured regional homogeneity ( $\mathrm{ReHo}$ ) and functional connectivity changes in resting-state brain networks pre- and post-VNS, in order to explore the functional mechanism of VNS therapy in resting-state networks anchored in the DMN and SN.

\section{Case report}

The study was approved by the medical ethics committee of the institutional review board of Beijing Tiantan Hospital, affiliated with Capital Medical University, and written informed consent was obtained from the patient and his parents to publish the patient's data and images. In addition, our study has been registered as ChiCTR-TRC-14005138 with the Chinese Clinical Trial Registry (ChiCTR) (www.chictr.org.cn).

A 17-year-old, right-handed male who started having seizures at the age of 12 years initially presented himself to our clinic. He reported focal epilepsy with right partial motor seizures and loss of consciousness lasting for 1-2 minutes, and occasional right lateralized myoclonic jerks (involving the right upper limb, lower limb, and facial and neck muscles) at a frequency of seven to eight times monthly. Occasionally, the patient reported having a headache or a sense of suppression in the chest before seizure onset. Moreover, this patient also had other epilepsy-attack forms, such as absence seizures and gelastic seizures, sometimes with automatism. These multiform grand mal status epilepticus attacks occurred almost daily. He had taken several kinds of AEDs with minimal benefit before receiving treatment in our epilepsy center.

Prior to treatment, routine physical and neurological exams were performed on the patient. Serial brain MRI examinations revealed a left parietal-occipital brain-region encephalomalacia focal signal alteration (Figure 1A). Electroencephalography (EEG) recordings were consistently characterized by interictal epileptiform discharges on the left parietal-occipital area and the anterior-middle temporal lobe. No clear EEG changes were correlated with epilepsy onset. First, we treated the patient with valproate and carbamazepine, in accordance with his medication history. After 1-year follow-up, the effects of antiepileptics for grand mal status epilepticus were obvious, but contributed nothing to his right lateralized myoclonic jerk seizures. At the same time, we reevaluated his MRI and EEG examinations, and found that his epilepsy condition was stable, and both MRI and EEG showed nonsignificant changes compared with the results from the previous year. Following evaluation of epilepsy symptoms, focal seizures, and a combination of MRI and EEG images, a left parietaloccipital lesion resection was performed on the patient (Figure 1B), with the neuropathology confirming the left parietal-occipital lesion was an encephalomalacia focus.

Unfortunately, the frequency of seizures increased rapidly after the resection surgery, reaching more than hourly frequency and occasionally with longer duration. Moreover, status epilepticus attacks occurred frequently. The epilepsy symptoms were controlled unsatisfactorily in the 1-year follow-up, despite the AEDs being replaced by oxcarbazepine, phenobarbital, and valproate. In addition, the patient had to withdraw from school because of the worsening condition of the illness. Afterward, he was evaluated for possible VNS therapy in our epilepsy center, in order to relieve the seizure condition. A VNS device (PINS

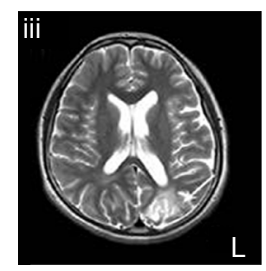

B

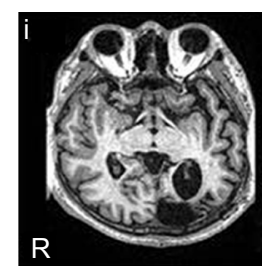

Postsurgery MRI

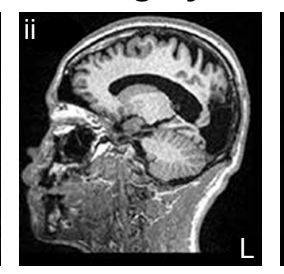

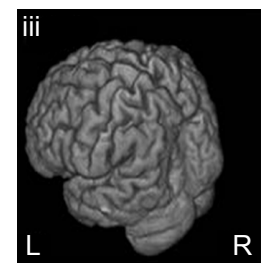

Presurgery MRI

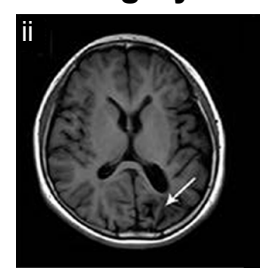

Figure I (A) MRI examination showing a left parietal-occipital encephalomalacia focal signal alteration in the patient's brain (arrow): sagittal $T_{1}$ MRI (i); axial $T_{1}$ MRI (ii); axial $T_{2}$-weighted MRI (iii). (B) MRI image of the patient's brain after left parietal-occipital lesion-resection surgery, showing that doubtful epileptic foci were completely removed: axial $T_{1}$ MRI (i); sagittal $T_{1}$ MRI (ii); three-dimensional MRI showing the resected cortex surface (iii).

Abbreviations: $M R I$, magnetic resonance imaging; $R$, right; $L$, left. 
A

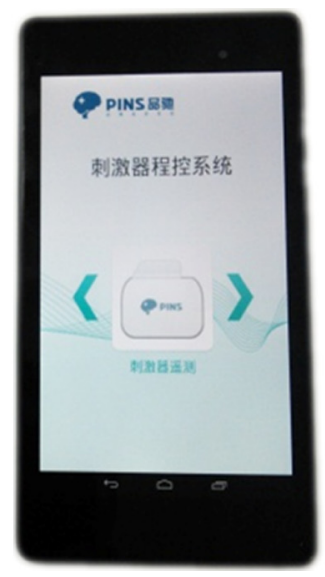

B

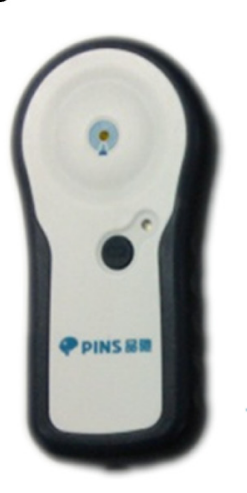

C

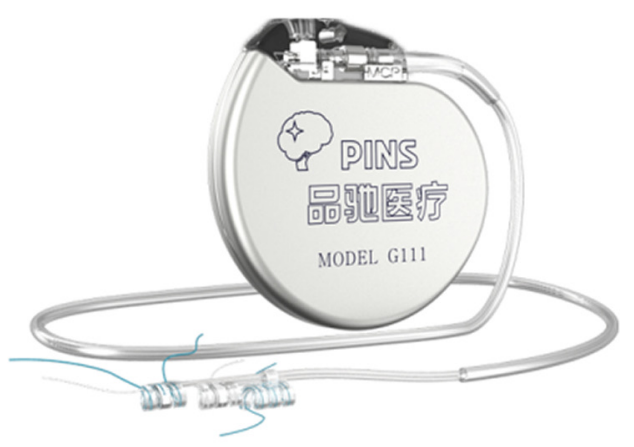

Figure 2 The vagus nerve-stimulation device used for implantation, obtained from PINS Medical (Beijing, People's Republic of China; http://www.pinsmedical.com/). Notes: (A) The handheld device is used for programming and to setup the stimulation parameters. (B) The communication-link device enables communication between the external handheld device and the internal implantable pulse generator. (C) The implantable pulse generator, accessory extension cord, and the leads which are fixed in the vagus nerve.

Medical, ${ }^{23,24}$ Figure 2) was subsequently implanted 1 year after the surgical resection. The stimulator was activated at $0.5 \mathrm{~mA}, 30 \mathrm{~Hz}, 500 \mu \mathrm{s}, 30$ seconds on, 5 minutes off 2 weeks after the day of surgery, and the amplitude was increased to $0.75 \mathrm{~mA}$ during the first month after implantation. During the first month, the patient became seizure-free, with a slight side effect of hoarseness, and was able to return to school. The amplitude was increased in steps of $0.25 \mathrm{~mA}$ until the ideal balance between seizure control and side effects was achieved. At the 6-month follow-up, the daytime seizures had disappeared almost completely, the frequency of lateralized myoclonic jerks and other forms of seizure were reduced by nearly two-thirds, and the seizure ictal time had been shortened to seconds. At the time of writing, the patient had been treated with VNS therapy for 6 months. The final stimulation parameters were current output $1.2 \mathrm{~mA}$, frequency $30 \mathrm{~Hz}$, pulse width $500 \mu \mathrm{s}, 30$ seconds signal on, 5 minutes signal off, magnet-triggered current $2 \mathrm{~mA}$, and stimulation duration 30 seconds. We will continue to collect this patient data every 2 months.

\section{Imaging methods and results}

A combination of fMRI in resting-state pre-VNS and 6-month post-VNS, high-resolution brain-structure images ( $T_{1}$-weighted 3-D magnetization-prepared rapid gradientecho sequences) and functional imaging (echo-planar imaging sequences) data obtained on a 1.5 T Siemens Magnetom Avanto MRI system (Siemens AG, Munich, Germany; scanning parameters detailed in Supplementary materials) were used for the current study. All data preprocessing was carried out using the SPM8 (http://www.fil.ion.ucl.ac.uk/spm) and DPARSF (http://www.restfmri.net/forum/DPARSF) toolkits.
All computational procedures (detailed in Supplementary materials) were carried out using the DPARSF default settings in the mode of "Calculate in original space (warp by DARTEL)".

As this was a single-subject study, we were unable to conduct group-level statistics, such as $t$-tests or analyses of variance. We thus adopted the statistical methods of Ibrahim et al. ${ }^{25}$ Briefly, we transformed all the calculation values to $Z$-scores by Fisher's transformation. Brain regions were then detected that showed $Z$-score changes greater than two standard-deviation differences between two scans, after which these brain regions were plotted. However, only positive $Z$-score changes were shown, as there have been conflicting results regarding negative changes in fMRI studies. ${ }^{26-28}$

The ReHo of the voxel at the center of the 27 nearest neighboring voxel clusters was calculated to reflect the regional functional connectivity changes. ${ }^{29}$ Large-scale functional connectivity analysis was performed by seeding the posterior cingulate cortex (PCC) and anterior cingulate cortex (ACC), as well as dominant nodes of the DMN and $\mathrm{SN}$, respectively. ${ }^{30,31}$ After seeding the PCC, Z-scores were increased in the DMN compared to pre-VNS therapy, and the suppressed DMN was reactivated. When the ACC was selected as a seed, Z-scores decreased greatly in the $\mathrm{SN}$, and overactivation of the SN was suppressed compared to pre-VNS scores. In addition, the links between the DMN and ACC were enhanced (Figure 3). However, compared to the pre-VNS-therapy ReHo maps (green in Figure 4), postVNS-therapy ReHo $Z$-scores showed marked increases in the left precentral gyrus, the medial prefrontal cortex (mPFC), and PCC regions, although there was no significant change in distribution regions. All other brain regions consistently 

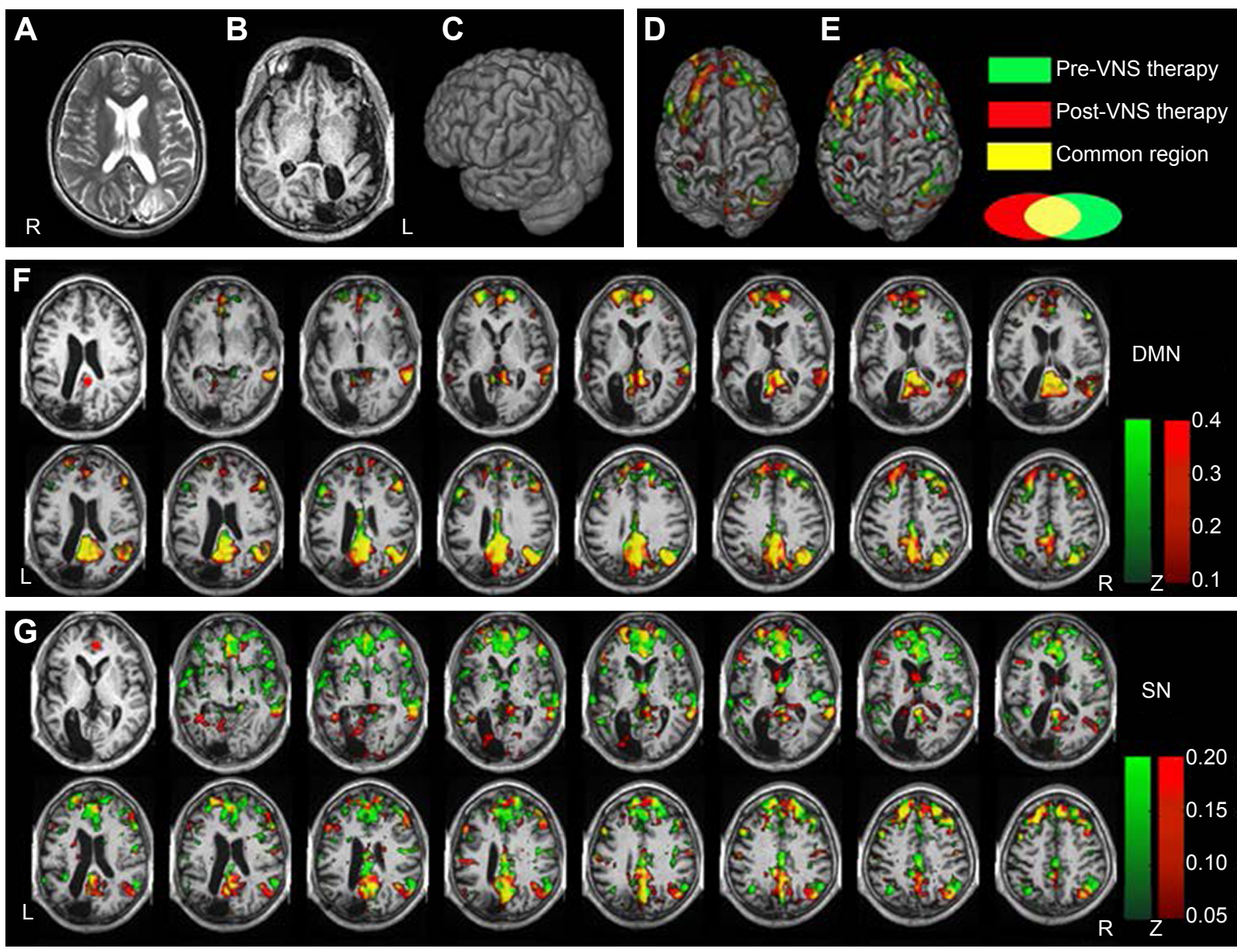

Figure 3 Seed-based FC was analyzed by anchoring the seed in the PCC and ACC, respectively.

Notes: Pre-VNS-therapy Z-score maps shown in green; post-VNS-therapy Z-score maps shown in red; yellow indicates common change regions. All brain-function maps were four voxel clusters corrected by the AFNI function 3dClustSim. (A) Axial $T_{2}$-weighted MRI before resection surgery. (B) Axial $T_{1}$ MRI after resection surgery. (C) 3-D view. (D) 3-D brain-surface view of FC changes by anchoring of PCC seed. (E) 3-D brain-surface view of FC changes by anchoring of ACC seed. (F) Compared to pre-VNS, Z-scores increased in the DMN, including the PCC, mFPC, and inferior parietal lobule, after receiving VNS therapy. Meanwhile, the DMN's links to some important brain regions were enhanced, including the superior frontal gyrus, right thalamus, ACC, posterior central gyrus, and anterior central gyrus, as shown in red. The connectives in the DMN were more powerful in post-VNS therapy compared to pre-VNS therapy. The PCC seed is marked with a red circle in the first slice on the left. The Z-score threshold was set at $Z>0$.I. (G) Compared to pre-VNS therapy, Z-scores decreased in the SN, including the bilateral ACC, caudate nuclei, insula, and dIPFC. The links among the DMN (PCC and inferior parietal lobule), posterior and anterior central gyrus, orbital frontal cortex, and ACC were also enhanced. The ACC seed is marked with a red circle in the leftmost slice. The $Z$-score threshold was set at $Z>0.05$.

Abbreviations: FC, functional connectivity; PCC, posterior cingulate cortex; ACC, anterior cingulate cortex; VNS, vagus nerve stimulation; AFNI, Analysis of Functional Neurolmages; MRI, magnetic resonance imaging; mFPC, medial prefrontal cortex; DMN, default-mode network; SN, salience network; dIPFC, dorsolateral PFC.

overlapped with the pre-VNS-therapy Z-score maps, as shown in Figure 4. In summary, after VNS therapy, ReHo $Z$-score maps were more focused within the DMN, and the visible difference in regional functional connectivity in the $\mathrm{SN}$ could not be reflected by the ReHo value.

These results point to the preliminary conclusions that VNS controls epileptic seizures, mainly through modulation of large-scale functional connectivity, and only mildly impacts regional functional connectivity. These conclusions are in agreement with the viewpoint that refractory epilepsy is a disease that is associated with a disrupted topological organization in large-scale brain networks, and not merely confined to regional changes. ${ }^{9-11}$

\section{Discussion}

According to a World Health Organization report, drugresistant epilepsy has been and continues to be a challenge to epilepsy treatment, especially to those who have undergone unsuccessful resection surgery and are not suitable for reoperation. ${ }^{32}$ Evidence-based guidelines published in 2013 updated the efficacy of VNS, indicating this treatment was associated with a $50 \%$ seizure reduction in 55\% of 470 epileptic children who underwent the procedure, while approximately $7 \%$ were reported to be seizure-free. ${ }^{5}$ Following initial small-sample studies by Ben-Menachem et $\mathrm{al}^{33}$ and Koutroumanidis et $\mathrm{al}^{34}$ on the effectiveness of VNS treatment on resection surgery-failure patients, there 


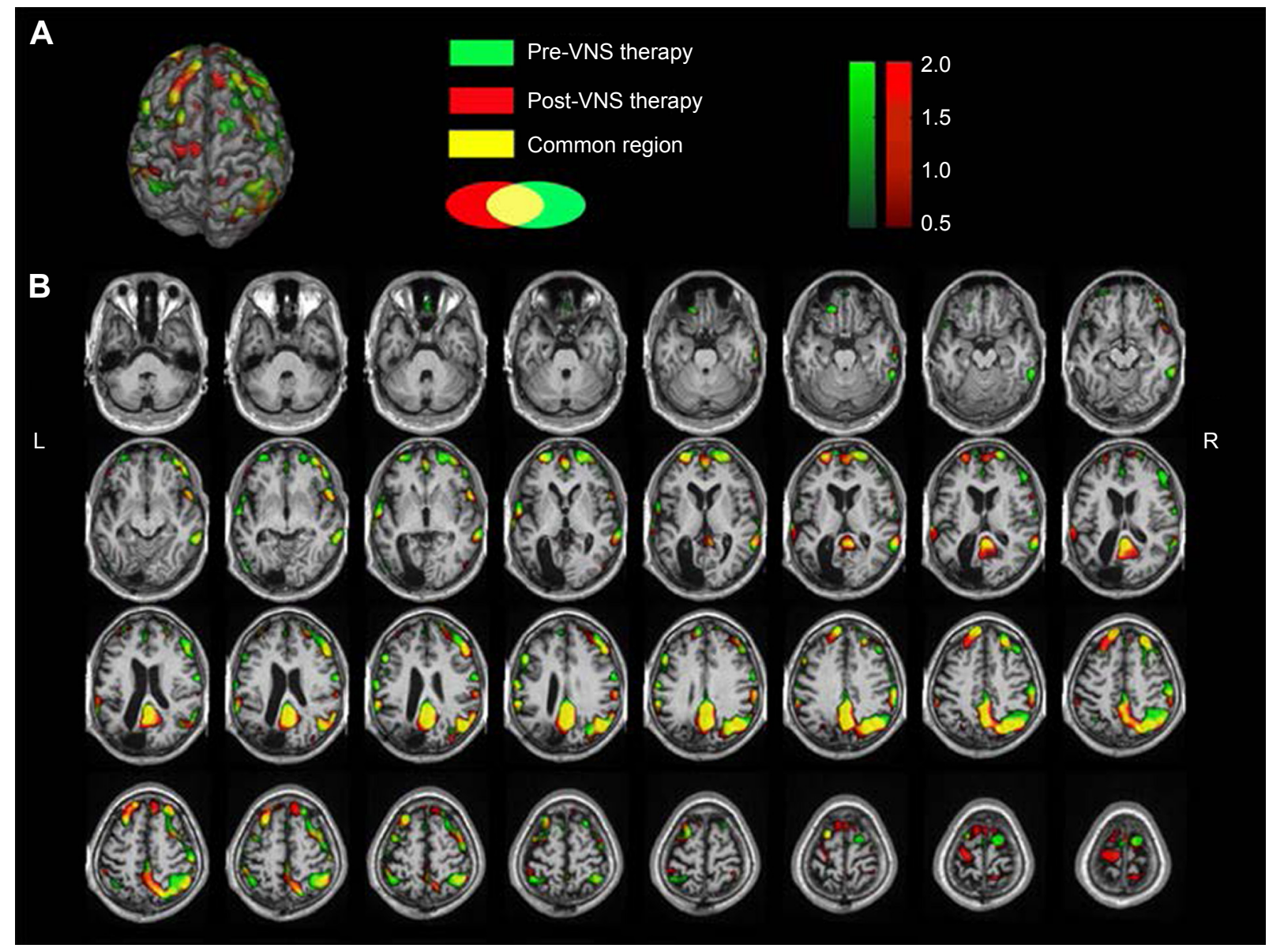

Figure 4 Z-values of ReHo between pre- and post-VNS therapy were overlapped after coregistering to common individual anatomical space.

Notes: Pre-VNS-therapy Z-score maps shown in green; post-VNS-therapy Z-scores maps shown in red; yellow indicates common changing regions. Compared to pre-VNS therapy (green), post-VNS therapy (red) ReHo greatly increased in the left precentral gyrus, mPFC, and PCC. After VNS therapy, ReHo Z-score maps were more focused on the DMN networks (red post-VNS network indicates stronger focus on the DMN region, while green pre-VNS network indicates weaker focus). All brain-function maps were four voxel clusters corrected by the AFNI function 3dClustSim. (A) 3-D brain image indicating ReHo changes. (B) Axial result maps. All ReHo results were set at a threshold of $Z>0.5$.

Abbreviations: ReHo, regional homogeneity; VNS, vagus nerve stimulation; mPFC, medial prefrontal cortex; PCC, posterior cingulate cortex; DMN, default-mode network; AFNI, Analysis of Functional Neurolmages.

have only been a few anecdotal reports on this area. In the current study, using both regional and global brain-network analyses of resting-state fMRI, we added to this body of data by exploring the mechanisms of VNS therapy on a patient with resection-surgery failure.

The prevailing view regarding VNS therapy has been that the therapy delivers electrical stimulation to the left cervical VN trunk, activating axons of afferent neurons, and subsequently initiates neuronal excitability throughout the central nervous system. ${ }^{35}$ However, whether there are fixed action targets involved in VNS therapy remain to be seen. Koutroumanidis et $\mathrm{al}^{34}$ suggested that the antiepileptic effect of VNS was not different with respect to the type of operation (anterior temporal lobectomy vs amygdalohippocampectomy), the side of operation, or the side of seizure onset. In their study, 16 patients with previous temporal and frontal resections were treated with VNS, and of those who had ineffective anterior temporal lobectomy, more than $50 \%$ experienced post-VNS seizure reduction without any clear postresection improvement, which was consistent with patients who had smaller resections (amygdalohippocampectomy). Indeed, one ipsilateral frontal lobectomy patient obtained $90 \%$ improvement in his daytime seizures. In a similar group of 12 patients, Kuba et al reported that two (one with a tumor) of six patients with right temporal resections responded favorably to VNS therapy, while three patients who had left temporal lobectomies showed a $20 \%-25 \%$ reduction in seizure frequency. ${ }^{36}$ In addition, one of three patients with right basal frontal resections showed $>75 \%$ seizure reduction, whereas the other two had a $75 \%$ reduction in seizure severity. These results indicate that frontal lobe resection can play a key role in the modulation of VNS therapy on epileptic activity, and that satisfactory seizure control tends to be largely associated with the frontal lobe. 
Indeed, most of the reported resected brain regions are the in temporal lobe, frontal lobe, hippocampus, or amygdaloid nucleus, while parietal-occipital resection cases have been rarely reported. ${ }^{33,34,36}$ Here, we report a case in which VNS therapy reduced the frequency of seizure by over $50 \%$ and at the same time increased the seizure-free length of time between these seizures.

Biswal et al first observed in $1995^{37}$ that resting-state fMRI could be used to identify networks within the brain by measuring low-frequency $(0.01-0.1 \mathrm{~Hz})$ fluctuations in BOLD signaling, which opened a new window into the brain. Recent neuroscience literature now has a wealth of information regarding brain networks and their roles in the pathogenesis of several diseases, such as epilepsy, schizophrenia, and Alzheimer's disease. ${ }^{30,38}$ The DMN and SN are two important discoveries in regard to the resting-state fMRI method based on the calculation of temporal correlations or coherence among BOLD signals. ${ }^{31,39-42}$ Deactivations at different levels in the DMN of epilepsy patients have been found, and such deactivations may explain the cognitive deficits and symptoms of declining working-memory ability among absence seizures, Lennox-Gastaut syndrome, and other forms of seizures. ${ }^{18,43}$ The SN was generally reported to be excessively activated in the ACC region of most epilepsy patients. ${ }^{44}$ Meanwhile, disorders of consciousness and attention could possibly be explained by the failure of the $\mathrm{SN}$ in integrating relevant internal and external stimuli, as well as a failure to interconnect with other functional networks that regulate behavior. However, the vast majority of existing studies and hypotheses are limited to the DMN or SN specifically, and interaction between the two has rarely been reported. Menon proposed a unifying triple-network model in psychopathology among the SN, DMN, and central executive network through anterior insula-mediated dynamic interactions with other large-scale brain networks. ${ }^{30}$ In line with this view, we assume a two-dimensional model in which VNS acts on intractable epilepsy by modulating the balance between the SN and DMN via an integral hub - the ACC. Our findings demonstrate that VNS mainly suppresses the SN's excessive activation and at the same time activates the suppressed DMN to achieve balance.

\section{Limitations}

Although the current study was limited by the fact that these findings were based on a single case study, this is only a preliminary attempt to look into the exact mechanisms of VNS therapy. Our two-dimensional model hypothesis will be tested in follow-up research with large-scale samples, and will identify more advanced and refined brain-network statistical analysis approaches, which will then be adapted in future work.

To this end, we have obtained the necessary ethical and management approvals given by the medical ethics committee of the institutional review board of Beijing Tiantan Hospital, affiliated with Capital Medical University, to carry out such a study.

\section{Acknowledgments}

This study was funded partly by the National Natural Science Foundation of China (grant number 81471315) and partly by the Beijing Municipal Commission of Science and Technology (grant number Z161100000516078).

\section{Disclosure}

The authors report no conflicts of interest in this work.

\section{References}

1. Ryvlin P, Cross JH, Rheims S. Epilepsy surgery in children and adults. Lancet Neurol. 2014;13(11):1114-1126.

2. Schuele SU, Luders HO. Intractable epilepsy: management and therapeutic alternatives. Lancet Neurol. 2008;7(6):514-524.

3. Theodore WH, Fisher RS. Brain stimulation for epilepsy. Lancet Neurol. 2004;3(2):111-118.

4. Berg AT, Vickrey BG, Testa FM, et al. How long does it take for epilepsy to become intractable? A prospective investigation. Ann Neurol. 2006;60(1):73-79.

5. Morris GL 3rd, Gloss D, Buchhalter J, Mack KJ, Nickels K, Harden C. Evidence-based guideline update: vagus nerve stimulation for the treatment of epilepsy: report of the Guideline Development Subcommittee of the American Academy of Neurology. Neurology. 2013;81(16): $1453-1459$.

6. Fisher RS, Handforth A. Reassessment: vagus nerve stimulation for epilepsy: a report of the Therapeutics and Technology Assessment Subcommittee of the American Academy of Neurology. Neurology. 1999;53(4):666-669.

7. Meng FG, Jia FM, Ren XH, et al. Vagus nerve stimulation for pediatric and adult patients with pharmaco-resistant epilepsy. Chin Med J (Engl). 2015;128(19):2599-2604.

8. Bodin C, Aubert S, Daquin G, et al. Responders to vagus nerve stimulation (VNS) in refractory epilepsy have reduced interictal cortical synchronicity on scalp EEG. Epilepsy Res. 2015;113:98-103.

9. Chassoux F, Artiges E, Semah F, et al. Determinants of brain metabolism changes in mesial temporal lobe epilepsy. Epilepsia. 2016;57(6): 907-919.

10. Engel J Jr, Thompson PM. Going beyond hippocampocentricity in the concept of mesial temporal lobe epilepsy. Epilepsia. 2012;53(1): $220-223$.

11. Richardson MP. Large scale brain models of epilepsy: dynamics meets connectomics. J Neurol Neurosurg Psychiatry. 2012;83(12): $1238-1248$.

12. Zhang Z, Liao W, Chen $\mathrm{H}$, et al. Altered functional-structural coupling of large-scale brain networks in idiopathic generalized epilepsy. Brain. 2011;134(Pt 10):2912-2928.

13. Liao W, Zhang Z, Mantini D, et al. Relationship between large-scale functional and structural covariance networks in idiopathic generalized epilepsy. Brain Connect. 2013;3(3):240-254.

14. An D, Dubeau F, Gotman J. BOLD responses related to focal spikes and widespread bilateral synchronous discharges generated in the frontal lobe. Epilepsia. 2015;56(3):366-374. 
15. Haneef Z, Lenartowicz A, Yeh HJ, Levin HS, Engel J Jr, Stern JM. Functional connectivity of hippocampal networks in temporal lobe epilepsy. Epilepsia. 2014;55(1):137-145.

16. Pizoli CE, Shah MN, Snyder AZ, et al. Resting-state activity in development and maintenance of normal brain function. Proc Natl Acad Sci US A. 2011;108(28):11638-11643.

17. Mesulam M. Defining neurocognitive networks in the BOLD new world of computed connectivity. Neuron. 2009;62(1):1-3.

18. Haneef Z, Lenartowicz A, Yeh HJ, Engel J Jr, Stern JM. Network analysis of the default mode network using functional connectivity MRI in temporal lobe epilepsy. J Vis Exp. 2014(90):e51442.

19. Broyd SJ, Demanuele C, Debener S, Helps SK, James CJ, SonugaBarke EJ. Default-mode brain dysfunction in mental disorders: a systematic review. Neurosci Biobehav Rev. 2009;33(3):279-296.

20. Anticevic A, Cole MW, Murray JD, Corlett PR, Wang XJ, Krystal JH The role of default network deactivation in cognition and disease. Trends Cogn Sci. 2012;16(12):584-592.

21. Chang WP, Shyu BC. Anterior cingulate epilepsy: mechanisms and modulation. Front Integr Neurosci. 2014;7:104

22. Luo C, Yang T, Tu S, et al. Altered intrinsic functional connectivity of the salience network in childhood absence epilepsy. $J$ Neurol Sci. 2014;339(1-2):189-195.

23. Liu HG, Ma Y, Zhang K, et al. Subthalamic deep brain stimulation with a new device in Parkinson's disease: an open-label trial. Neuromodulation. 2013;16(3):212-218.

24. Jia F, Guo Y, Wan S, et al. Variable frequency stimulation of subthalamic nucleus for freezing of gait in Parkinson's disease. Parkinsonism Relat Disord. 2015;21(12):1471-1472.

25. Ibrahim GM, Morgan BR, Smith ML, et al. Thalamocortical connectivity is enhanced following functional hemispherotomy for intractable lateralized epilepsy. Epilepsy Behav. 2015;51:281-285.

26. Scholvinck ML, Maier A, Ye FQ, Duyn JH, Leopold DA. Neural basis of global resting-state fMRI activity. Proc Natl Acad Sci U S A. 2010;107(22):10238-10243.

27. Murphy K, Birn RM, Handwerker DA, Jones TB, Bandettini PA. The impact of global signal regression on resting state correlations: are anticorrelated networks introduced? Neuroimage. 2009;44(3):893-905.

28. Fox MD, Zhang D, Snyder AZ, Raichle ME. The global signal and observed anticorrelated resting state brain networks. J Neurophysiol. 2009;101(6):3270-3283.

29. Zang Y, Jiang T, Lu Y, He Y, Tian L. Regional homogeneity approach to fMRI data analysis. Neuroimage. 2004;22(1):394-400.
30. Menon V. Large-scale brain networks and psychopathology: a unifying triple network model. Trends Cogn Sci. 2011;15(10):483-506.

31. Zhang D, Raichle ME. Disease and the brain's dark energy. Nature Rev Neurol. 2010;6(1):15-28.

32. Moshé SL, Perucca E, Ryvlin P, Tomson T. Epilepsy: new advances. Lancet. 2015;385(9971):884-898.

33. Ben-Menachem E, Hellström K, Waldton C, Augustinsson LE. Evaluation of refractory epilepsy treated with vagus nerve stimulation for up to 5 years. Neurology. 1999;52(6):1265-1267.

34. Koutroumanidis M, Binnie CD, Hennessy MJ, et al. VNS in patients with previous unsuccessful resective epilepsy surgery: antiepileptic and psychotropic effects. Acta Neurol Scand. 2003;107(2):117-121.

35. Attenello F, Amar AP, Liu C, Apuzzo ML. Theoretical basis of vagus nerve stimulation. Prog Neurol Surg. 2015;29:20-28.

36. Kuba R, Brazdil M, Kalina M, et al. Vagus nerve stimulation: longitudinal follow-up of patients treated for 5 years. Seizure. 2009;18(4): 269-274.

37. Biswal B, Yetkin FZ, Haughton VM, Hyde JS. Functional connectivity in the motor cortex of resting human brain using echo-planar MRI Magn Reson Med. 1995;34(4):537-541.

38. Sheline YI, Raichle ME. Resting state functional connectivity in preclinical Alzheimer's disease. Biol Psychiatry. 2013;74(5):340-347.

39. Greicius MD, Krasnow B, Reiss AL, Menon V. Functional connectivity in the resting brain: a network analysis of the default mode hypothesis. Proc Natl Acad Sci U S A. 2003;100(1):253-258.

40. Greicius MD, Srivastava G, Reiss AL, Menon V. Default-mode network activity distinguishes Alzheimer's disease from healthy aging: evidence from functional MRI. Proc Natl Acad Sci US A. 2004;101(13): 4637-4642.

41. Menon V, Uddin LQ. Saliency, switching, attention and control: a network model of insula function. Brain Struct Funct. 2010;214(5-6): 655-667.

42. Seeley WW, Menon V, Schatzberg AF, et al. Dissociable intrinsic connectivity networks for salience processing and executive control. J Neurosci. 2007;27(9):2349-2356.

43. Cataldi M, Avoli M, de Villers-Sidani E. Resting state networks in temporal lobe epilepsy. Epilepsia. 2013;54(12):2048-2059.

44. Yang T, Fang Z, Ren J, et al. Altered spontaneous activity in treatmentnaive childhood absence epilepsy revealed by regional homogeneity. J Neurol Sci. 2014;340(1-2):58-62. 


\section{Supplementary materials Imaging methods}

High-resolution brain-structure images were acquired using $T_{1}$-weighted, sagittal 3-D magnetization-prepared rapid gradient-echo sequences with the following parameters: repetition time $\left(\mathrm{T}_{\mathrm{R}}\right)$ /echo time $\left(\mathrm{T}_{\mathrm{E}}\right)$ /inversion time $=2,400 / 3.92 / 1,000 \mathrm{~ms}$, flip angle (FA) $8^{\circ}$, field of view (FOV) $256 \times 256 \times 160 \mathrm{~mm}$, voxel size $1 \times 1 \times 1 \mathrm{~mm}^{3}$, slice thickness $1 \mathrm{~mm}$, and 160 sagittal slices. Functional images were collected axially using an echo-planar imaging (EPI) sequence with the following settings: $T_{R} / T_{E}=2,000 / 40 \mathrm{~ms}$, FA $80^{\circ}$, FOV $240 \mathrm{~mm} 240 \times 115 \mathrm{~mm}$, axial slices 24 , thickness $4 \mathrm{~mm}$, spacing between slices $4.8 \mathrm{~mm}$, voxel size $3.8 \times 3.8 \times 4 \mathrm{~mm}^{3}$, bandwidth $1,735 \mathrm{~Hz} /$ pixel, volume 200 . All data were obtained on a $1.5 \mathrm{~T}$ Magnetom Avanto magnetic resonance imaging (MRI) system (Siemens AG, Munich, Germany) prior to vagus nerve stimulation (VNS) implantation and 6 months after VNS activation. Prior to the restingstate functional MRI (fMRI) data scan, the patient was instructed to keep his eyes closed, relax but not fall asleep, and move as little as possible during scanning. The VNS had remained off for 3 minutes before MRI scanning for the sake of safety.

All data preprocessing was carried out using SPM8 (http://www.fil.ion.ucl.ac.uk/spm) and DPARSF (http://www.restfmri.net/forum/DPARSF) toolkits. Data preprocessing included discarding the first ten volumes of EPI, slice-timing correction, head-motion correction, coregistration of EPI to high-resolution $T_{1}$-weighted images, and coregistration between pre- and postimage data. Spatially coregistered images were then resampled to an isotropic voxel size of $3 \times 3 \times 3 \mathrm{~mm}^{3}$ and smoothed with a $6 \mathrm{~mm}$ fullwidth half-maximum Gaussian kernel. Linear detrending and temporal band pass $(0.01-0.08 \mathrm{~Hz})$ filtering were performed, in order to remove low-frequency drifts and physiologic highfrequency noise. In addition, global mean signal, six motion parameters, cerebrospinal fluid signal, and white-matter signal were regressed out to reduce the effects of head motion and other physiological fluctuations. Finally, Z-score calculations of regional homogeneity ( $\mathrm{ReHo}$ ) and functional connectivity (FC) were completed and statistical analyses performed. We calculated individual FC based on two seeds to measure global

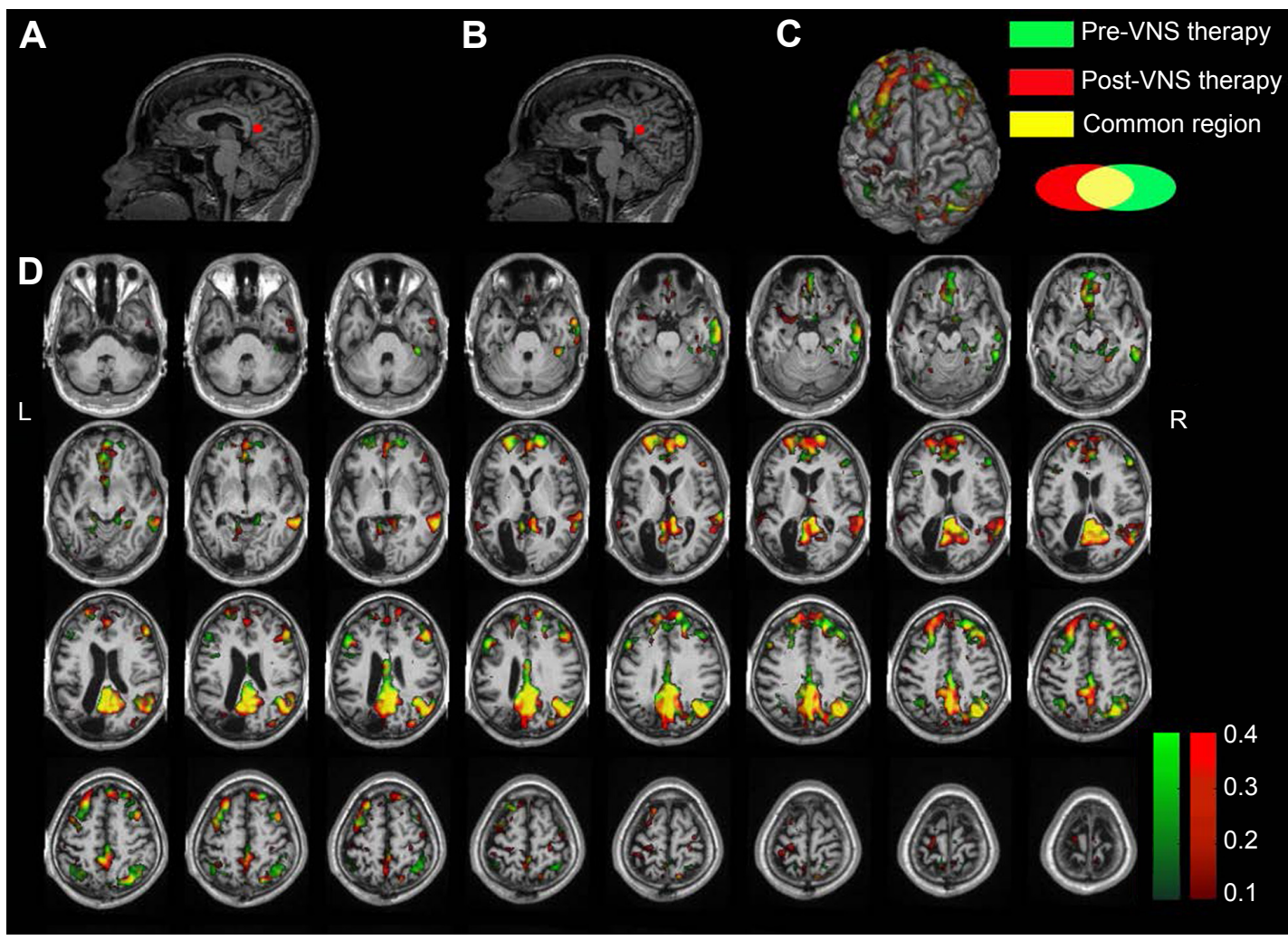

Figure SI Seed-based analysis anchoring the ROI in the PCC.

Notes: Compared to pre-VNS therapy, Z-scores increased in the DMN, including the PCC, mFPC, and inferior parietal lobule post-VNS therapy. The DMN links to several important brain regions were enhanced, including the superior frontal gyrus, right thalamus, ACC, posterior central gyrus, and anterior central gyrus, as shown in red. The connectives in the DMN post-VNS therapy were more powerful than pre-VNS therapy. (A) Anatomical images showing the PCC seed selected pre-VNS therapy. (B) Anatomical images showing the PCC seed selected post-VNS therapy. (C) 3-D brain rendering showing brain-surface changes. (D) Axial result maps. All results were set at a threshold of $Z>0$.I.

Abbreviations: ROI, region of interest; PCC, posterior cingulate cortex; VNS, vagus nerve stimulation; DMN, default-mode network; mFPC, medial prefrontal cortex; ACC, anterior cingulate cortex; L, left; $R$, right. 
feature changes. ${ }^{1,2}$ All computational procedures were carried out using DPARSF default settings in the mode of "Calculate in original space (warp by DARTEL)". FC analysis was performed by seeding the posterior cingulate cortex (PCC) and anterior cingulate cortex (ACC) and dominant nodes of the default-mode network (DMN) and salience network (SN), after which the mean time series of a manually drawn region of interest (ROI) in the PCC and ACC were correlated with the time series of all voxels in the brain. Seeds were handdrawn, as shown in Figure S1A and B. Data-result maps were displayed by MRIcroGL (http://www.mccauslandcenter. sc.edu/mricrogl), 3-D medical imaging software.

The similarity of the time series within a cluster was measured based on the ReHo method. ${ }^{3}$ The ReHo of the voxel at the center of the 27 nearest neighboring voxels cluster was calculated by Kendall's coefficient-of-concordance algorithm by REST software (http://www.restfmri.net).

\section{Results}

As this was a single-subject study, we were unable to conduct group-level statistics, such as $t$-tests or analyses of variance, we thus transformed all values to $Z$-scores using Fisher's transformation. Additionally, using the distribution of $Z$-scoredifference maps derived by coregistering the before-and-after images, we identified regions that showed obvious differences from the brain-region distribution. However, we only showed positive $Z$-score changes, as there have been conflicting results regarding negative changes in fMRI studies. ${ }^{4-6}$

\section{Seed-based functional connectivity}

Seed-based ROI analysis was performed with REST software. fMRI data were filtered using a band-pass filter $(0.01-0.08 \mathrm{~Hz})$ to reduce low-frequency drift and high-frequency noise. Two seeds selected for connectivity analysis were the PCC (Figure S1A and B) and the ACC (Figure S2A and B), in which the former seed was a dominant central node of the DMN, and the latter always played a critical role in the SN. When the PCC was seeded, $Z$-scores increased in the DMN, including the PCC, mFPC, and inferior parietal lobule (Figure S1). What is more, the DMN's links to some important brain regions were enhanced, including the superior frontal gyrus, right thalamus, ACC, posterior central

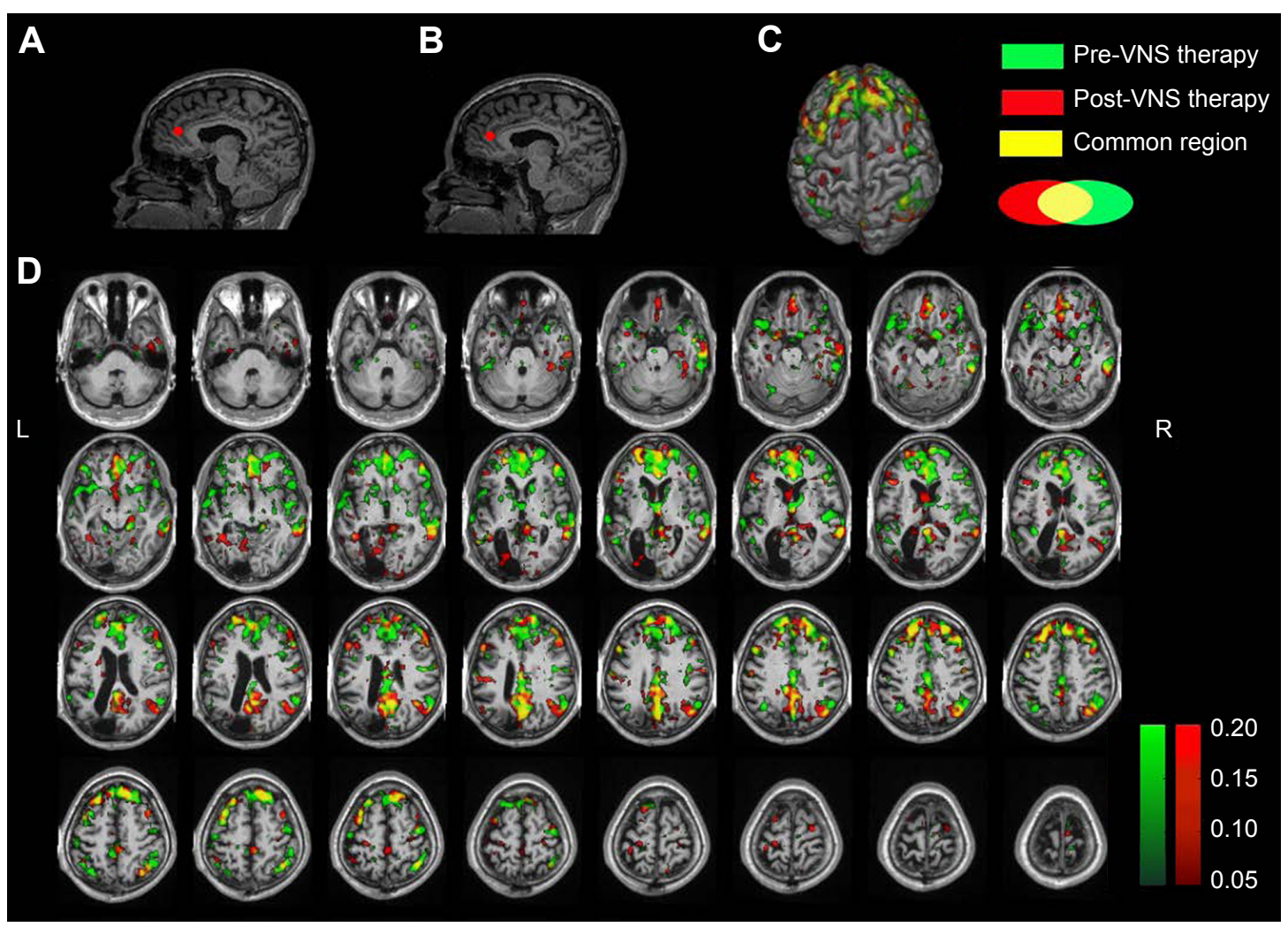

Figure S2 Seed-based analysis anchoring the ROI in the ACC.

Notes: Compared to pre-VNS therapy, Z-scores greatly decreased in the bilateral ACC, caudate nuclei, insula, dIPFC, and medial frontal cortex, and the links among the DMN (PCC, inferior parietal lobule), posterior and anterior central gyrus, orbital frontal cortex, and ACC were enhanced. As shown, the connectives among nodes in the DMN were enhanced, but those in the SN greatly decreased. (A) Anatomical images showing the ACC seed selected pre-VNS therapy. (B) Anatomical images showing the ACC seed selected post-VNS therapy. (C) 3-D brain rendering showing the changing brain surface. (D) Axial result maps. All results were set at a threshold of $Z>0.05$.

Abbreviations: ROI, region of interest; ACC, anterior cingulate gyrus; VNS, vagus nerve stimulation; dIPFC, dorsolateral prefrontal cortex; PCC, posterior cingulate gyrus; DMN, default-mode network; SN, salience network; L, left; R, right. 


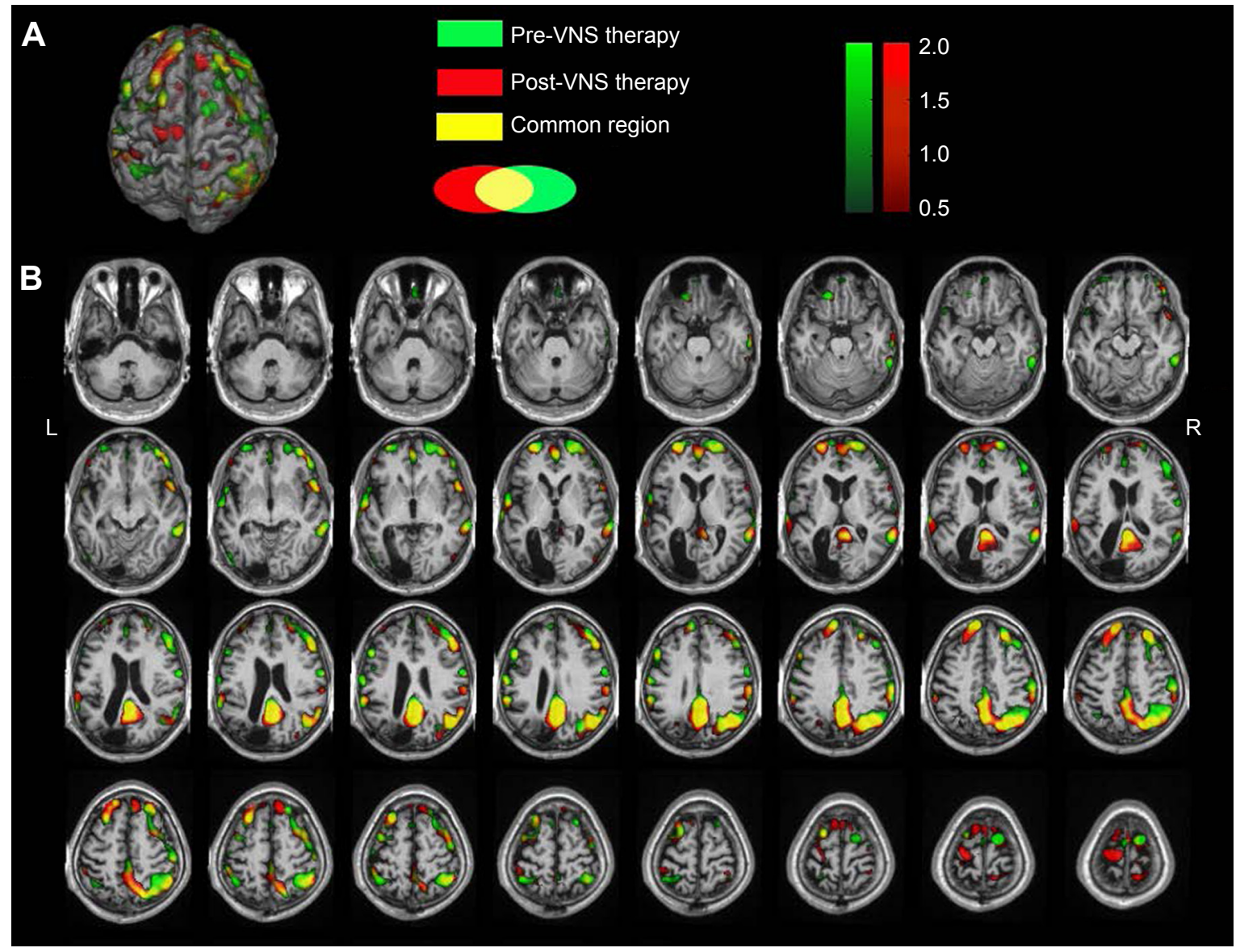

Figure S3 Overlap comparison of ReHo Z-values between pre- and post-VNS therapy after coregistering to common individual anatomical space.

Notes: Pre-VNS-therapy Z-score maps shown in green; post-VNS-therapy Z-score maps shown in red; yellow shows common changing regions. Compared to pre-VNS therapy (green), post-VNS therapy (red) ReHo values greatly increased in the left precentral gyrus, mPFC, and PCC regions. After VNS therapy, ReHo Z-score maps were more focused on the DMN (red represents a greater focus on the DMN region, while green represents areas of less DMN focus). (A) 3-D brain rendering showing changes in ReHo values. (B) Axial result maps. All ReHo results were set at a threshold of $Z>0.5$.

Abbreviations: ReHo, regional homogeneity; VNS, vagus nerve stimulation; mPFC, medial prefrontal cortex; PCC, posterior cingulate cortex; DMN, default-mode network; L, left, R, right.

gyrus, and anterior central gyrus, as shown in Figure S1. When the ACC was selected as a seed, the difference of FC in the SN could be clearly seen (Figure S2). Compared to pre-VNS therapy, Z-scores decreased in the bilateral ACC, caudate nuclei, insula, dorsolateral prefrontal cortex and medial frontal cortex, and the links among the DMN (PCC, inferior parietal lobule), posterior and anterior central gyrus, orbit frontal cortex, and ACC were enhanced.

\section{ReHo}

Compared to pre-VNS-therapy ReHo maps, post-VNS therapy ReHo showed obvious increases in the left precentral gyrus and medial prefrontal cortex, while PCC regions showed increased $Z$-scores. No significant changes in distribution regions were found. All other brain regions consistently overlapped with pre-VNS-therapy Z-score maps (Figure S3).
Following VNS therapy, ReHo Z-score maps were more focused on the DMN. The visible difference in regional FC in the $\mathrm{SN}$ was not reflected by ReHo value.

\section{References}

1. Ibrahim GM, Morgan BR, Smith ML, et al. Thalamocortical connectivity is enhanced following functional hemispherotomy for intractable lateralized epilepsy. Epilepsy Behav. 2015;51:281-285.

2. Menon V. Large-scale brain networks and psychopathology: a unifying triple network model. Trends Cogn Sci. 2011;15(10):483-506.

3. Zang Y, Jiang T, Lu Y, He Y, Tian L. Regional homogeneity approach to fMRI data analysis. Neuroimage. 2004;22(1):394-400.

4. Scholvinck ML, Maier A, Ye FQ, Duyn JH, Leopold DA. Neural basis of global resting-state fMRI activity. Proc Natl Acad Sci U S A. 2010;107(22):10238-10243.

5. Murphy K, Birn RM, Handwerker DA, Jones TB, Bandettini PA. The impact of global signal regression on resting state correlations: are anticorrelated networks introduced? Neuroimage. 2009;44(3):893-905.

6. Fox MD, Zhang D, Snyder AZ, Raichle ME. The global signal and observed anticorrelated resting state brain networks. $J$ Neurophysiol. 2009;101(6):3270-3283 
Neuropsychiatric Disease and Treatment

Dovepress

\section{Publish your work in this journal}

Neuropsychiatric Disease and Treatment is an international, peerreviewed journal of clinical therapeutics and pharmacology focusing on concise rapid reporting of clinical or pre-clinical studies on a range of neuropsychiatric and neurological disorders. This journal is indexed on PubMed Central, the 'PsycINFO' database and CAS,

and is the official journal of The International Neuropsychiatric Association (INA). The manuscript management system is completely online and includes a very quick and fair peer-review system, which is all easy to use. Visit http://www.dovepress.com/testimonials.php to read real quotes from published authors.

Submit your manuscript here: http://www.dovepress.com/neuropsychiatric-disease-and-treatment-journal 